\title{
Conserved masses in GHS Einstein and string black holes and consistent thermodynamics
}

\author{
K. C. K. Chan, ${ }^{*}$ J. D. E. Creighton, ${ }^{\dagger}$ and R. B. Mann ${ }^{\ddagger}$ \\ Dept. of Physics, University of Waterloo, Waterloo, Ontario, Canada. N2L 3G1
}

(29 April, 1996)

\begin{abstract}
We analyze the relationship between quasilocal masses calculated for solutions of conformally related theories. We show that the ADM mass of a static, spherically symmetric solution is conformally invariant (up to a constant factor) only if the background action functional is conformally invariant. Thus, the requirement of conformal invariance places restrictions on the choice of reference spacetimes. We calculate the mass of the black hole solutions obtained by Garfinkle, Horowitz, and Strominger (GHS) for both the string and the Einstein metrics. In addition, the quasilocal thermodynamic quantities in the string metrics are computed and discussed.
\end{abstract}

*Electronic address: kckchan@avatar.uwaterloo.ca

${ }^{\dagger}$ Electronic address: jolien@avatar.uwaterloo.ca

$\ddagger$ Electronic address: mann@avatar.uwaterloo.ca 


\section{INTRODUCTION}

General considerations regarding the structure of quantum gravity suggest that general relativity may require some modification, even at energies considerably lower than the Planck scale. Dilatonic gravity theories have recently played a prominent role in these considerations since dilaton gravity emerges as the low-energy effective field theory limit of string theories. The general form of the action of such theories is

$$
I=\int_{\mathcal{M}}{ }^{n} \boldsymbol{\epsilon}\left(D(\phi) R+H(\phi)(\nabla \phi)^{2}+V(\phi)\right)+I_{\mathrm{M}}
$$

where $\mathcal{M}$ is a $n$-dimensional manifold with volume $n$-form ${ }^{n} \boldsymbol{\epsilon} ; D, H$, and $V$ are arbitrary functions of the dilaton field $\phi ; R$ is the Ricci curvature scalar; and $I_{\mathrm{M}}$ is the matter action. In general, the matter action will be a functional of the dilaton, the metric, and whatever matter fields are relevant to the system of interest.

Specification of $D, H$, and $V$ is tantamount to the specification of a given dilaton theory of gravity in the absence of coupling to matter. When $D$ does not depend upon the dilaton, the theory represents general relativity with a minimally coupled scalar field. However, we may always perform a conformal tranformation,

$$
g_{a b}=\Omega^{2}(\phi) g_{a b}^{\mathrm{E}},
$$

that will give a metric that is a solution to the field equations obtained from the action of eq. (11.1) with $D(\phi) \propto \Omega^{2-n}(\phi)$. In eq. (1.2), the metric representing a solution to Einstein's general relativity is denoted by $g_{a b}^{\mathrm{E}}$.

It is often assumed that physical quantities, such as the mass and thermodynamic variables, should be the same for conformally related solutions. For example, in a previous paper [1], Garfinkle, Horowitz, and Strominger (GHS) derived the (3+1)-dimensional magnetically (or electrically) charged string black hole solutions (for which $H / 4=D \propto \exp (-2 \phi)$ ) in terms of the Einstein metric by performing the conformal tranformation

$$
g_{a b}^{\mathrm{S}}=\mathrm{e}^{2 \phi} g_{a b}^{\mathrm{E}} .
$$

By assuming that a conformal transformation would preserve physical quantities [2] such as the ADM mass, they claimed that the ADM mass in the string metric is the same as the one in the Einstein metric."]

In this paper, we show that the situation is more subtle. Specifically, we show that the quasilocal generalization of the ADM mass for systems of finite size [3, [4] is not generally conformally invariant. This result is a consequence of the lack of conformal invariance of the background action functional that specifies a reference spacetime. The mass is conformally invariant only if the background action functional, which specifies the reference spacetime, is required to be conformally invariant. Even then the ADM mass may differ between conformally related theories if the conformal transformation does not approach unity

\footnotetext{
${ }^{1}$ By ADM mass, we mean the value of the Hamiltonian conjugate to unit time translations at spacelike infinity for asymptotically flat spacetimes.
} 
at spacelike infinity, but this difference is just a constant factor due to a trivial rescaling of the time coordinate. As an illustration, we use the GHS string black hole solutions: We calculate the ADM mass directly from the string action using the aforementioned quasilocal formalism [四] as well as from the Einstein action obtained via the conformal transformation of eq. (1.3). We show that the ADM masses are the same, up to a constant factor, when a suitable background action functional is chosen. In addition, we shall compute the thermodynamic quantities relevant to the string metric and show that these are consistent with the first law of thermodynamics. It should be noted that the validity of the first law of thermodynamics does not depend on the choice of the reference spacetime, although the individual thermodynamic variables do.

In Sec. II, we discuss the general circumstance under which conformal invariance of the mass can be expected to hold, and we construct a conformally invariant expression for the quasilocal mass for a static, spherically symmetric spacetime. We review the definition of the quasilocal thermodynamic variables in Sec. III]. The example of the GHS solution is explored in Sec. IV] where we compute the ADM mass in both the string and the Einstein forms of the theory. We also calculate the thermodynamic variables for both the electric and the magnetic string black hole solutions, and explore their asymptotically large-system-size behaviour. In the case of the magnetic string solution, we need to modify the quasilocal formalism to avoid the Dirac string singularity in the Maxwell potential. Finally, we summarize our results in the concluding section.

\section{CONFORMAL TRANSFORMATIONS AND QUASILOCAL MASS}

We wish to obtain a notion of mass for solutions of theories of dilaton gravity that has the property that is invariant under conformal transformations of the solutions. That is, given two solutions of conformally related theories that are themselves conformally related, we want to have a definition of mass that yields the same value for both solutions. In this section we shall address some of the problems involved in the construction of such a mass. Let us first summarize what it means for two theories to be conformally related.

\section{A. Conformally Related Theories}

Consider two theories of dilaton gravity with metrics $g_{a b}$ and $\tilde{g}_{a b}$, dilaton fields $\phi$ and $\tilde{\phi}$, and actions

$$
\begin{gathered}
I=\int_{\mathcal{M}}{ }^{n} \boldsymbol{\epsilon}\left(D(\phi) R+H(\phi)(\nabla \phi)^{2}+V(\phi)\right) \\
-2 \int_{\partial \mathcal{M}}{ }^{(n-1)} \boldsymbol{\epsilon} D(\phi) \Theta-I_{0}
\end{gathered}
$$

and

$$
\begin{gathered}
\tilde{I}=\int_{\mathcal{M}}{ }^{n} \tilde{\boldsymbol{\epsilon}}\left(\tilde{D}(\tilde{\phi}) \tilde{R}+\tilde{H}(\tilde{\phi})(\nabla \tilde{\phi})^{2}+\tilde{V}(\tilde{\phi})\right) \\
-2 \int_{\partial \mathcal{M}}{ }^{(n-1)} \tilde{\boldsymbol{\epsilon}} \tilde{D}(\tilde{\phi}) \tilde{\Theta}-\tilde{I}_{0}
\end{gathered}
$$


Here, $\partial \mathcal{M}$ is the boundary of $\mathcal{M}$ with normal $n^{a}$, induced metric $\gamma_{a b}=g_{a b}-n_{a} n_{b}$, extrinsic curvature $\Theta_{a b}=-\frac{1}{2} £_{n} \gamma_{a b}$, and volume element ${ }^{(n-1)} \boldsymbol{\epsilon}=n \cdot{ }^{n} \boldsymbol{\epsilon}$. The background action $I_{0}$ is a functional of the fields on $\partial \mathcal{M}$ alone; the background action does not contribute to the field equations, but it specifies a reference spacetime that effectively defines zero mass. The quantities with a tilde are defined similarly.

We wish to determine the relationship between the functions of the dilaton under the requirement that the two theories are related by the conformal tranformation

$$
\tilde{g}_{a b}=\Omega^{2}(\phi) g_{a b} \quad \text { and } \quad \tilde{\phi}=\Upsilon(\phi)
$$

Under this transformation we have

$$
\begin{gathered}
{ }^{n} \tilde{\boldsymbol{\epsilon}} \tilde{D}(\tilde{\phi}) \tilde{R}={ }^{n} \boldsymbol{\epsilon} \Omega^{n-2} \tilde{D}(\tilde{\phi})\left(R-2(n-1) \nabla^{2} \log \Omega\right. \\
\left.-(n-2)(n-1)(\nabla \Omega)^{2}\right)
\end{gathered}
$$

and

$$
\begin{array}{r}
{ }^{(n-1)} \tilde{\boldsymbol{\epsilon}} \tilde{D}(\tilde{\phi}) \tilde{\Theta}={ }^{(n-1)} \boldsymbol{\epsilon} \Omega^{n-1} \tilde{D}(\tilde{\phi})\left(\Omega^{-1} \Theta\right. \\
\left.-(n-1) n^{a} \nabla_{a} \log \Omega\right) .
\end{array}
$$

It is clear that a necessary condition for the actions of Eqs. 2.1) to transform each other is $\tilde{D}(\tilde{\phi})=\Omega^{2-n}(\phi) D(\phi)$. With this relation, we find

$$
\begin{aligned}
& \int_{\tilde{\mathcal{M}}}{ }^{n} \tilde{\boldsymbol{\epsilon}} \tilde{D}(\tilde{\phi}) \tilde{R}-2 \int_{\partial \tilde{\mathcal{M}}}{ }^{(n-1)} \tilde{\boldsymbol{\epsilon}} \tilde{D}(\tilde{\phi}) \tilde{\Theta} \\
& =\int_{\mathcal{M}}{ }^{n} \boldsymbol{\epsilon}(D(\phi) R+\cdots)-2 \int_{\partial \mathcal{M}}{ }^{\left({ }^{n-1}\right)} \boldsymbol{\epsilon} D(\phi) \Theta
\end{aligned}
$$

where we have integrated by parts on the second last term of the right hand side of eq. (2.3). The boundary terms so produced are exactly cancelled by the $\Omega$-dependent terms from the extrinsic curvatures in eq. (2.4).

The terms in the ellipsis of eq. (2.5) are quadratic in $\nabla \log \Omega$ and, therefore, are quadratic in $\nabla \phi$. Upon examination of these terms, it is straightforward to show that equivalence between the two theories further requires

$$
\left(\frac{\tilde{H}}{\tilde{D}}-\frac{n-1}{n-2}\left(\frac{\tilde{D}^{\prime}}{\tilde{D}}\right)^{2}\right)\left(\Upsilon^{\prime}\right)^{2}=\left(\frac{H}{D}-\frac{n-1}{n-2}\left(\frac{D^{\prime}}{D}\right)^{2}\right)
$$

and

$$
\tilde{D}^{n /(2-n)} \tilde{V}=D^{n /(2-n)} V
$$

where the prime denotes a derivative with respect to the functional argument. (For the $n=2$ version of these relations, see Ref. [5].) Hence, the actions of Eqs. (2.1) transform into one another under the transformation (2.2), provided the reference actions appropriately transform into each other. 


\section{B. Quasilocal Mass}

Because conserved quantities such as the mass are only well defined with reference to the background action $I_{0}$, the conserved quantities defined in a theory given by the action of eq. (2.1a) will be the same as those defined in a theory given by the action of eq. (2.1b) only if the background action functionals also transform into one another under the transformation (2.2). Then the conformal transformation is just a field redefinition under which physical quantities should be invariant. However, if the background action functionals do not tranform into each other, then the field equations will still be conformally related, but the conserved quantities (and thermodynamic variables) calculated from the solutions will not agree in general.

Thus, it is important to select the appropriate background action functionals. Clearly the choice $I_{0}=0$ has the appropriate tranformation property and is insensitive to any special symmetries a given solution may have. However, the mass (and other conserved quantities) will not generally have a finite limit as the quasilocal system expands to infinite size.

Consider as another choice the background action functionals

$$
I_{0}=-2 \int_{\mathcal{T}} N \boldsymbol{d} t \wedge^{(n-2)} \boldsymbol{\epsilon} D(\phi) k_{\mathrm{flat}}
$$

and

$$
\tilde{I}_{0}=-2 \int_{\mathcal{T}} \tilde{N} \boldsymbol{d} t \wedge^{(n-2)} \tilde{\boldsymbol{\epsilon}} \tilde{D}(\tilde{\phi}) \tilde{k}_{\mathrm{flat}} .
$$

Here, the timelike boundary $\mathcal{T}$ is chosen to contain the orbits of the timelike Killing vector $(\partial / \partial t)^{a}$. The lapse function $N=\left(u^{a} \nabla_{a} t\right)^{-1}$ measures the normalization of the unit normal $u^{a}$ to the spacelike surfaces $\Sigma_{t}$ of constant $t$. The quantity $k_{\text {flat }}$ is the trace of the extrinsic curvature of the $(n-2)$-surface $\mathcal{T} \cap \Sigma_{t}$, together with its geometry $\sigma_{a b}$, in flat Euclidean space of dimension $n-1$. Similar definitions apply to the variables $\tilde{N}$ and $\tilde{k}_{\text {flat }}$. Conserved quantities will remain invariant under (2.2) provided (2.8a) transforms into (2.8b) under this transformation.

Let us consider a specific example: Suppose we are interested in a static, spherically symmetric (SSS) solution to a theory of dilaton gravity given by eq. 2.1a) of the form

$$
d s^{2}=-N^{2}(r) d t^{2}+\frac{d r^{2}}{f^{2}(r)}+r^{2} d \omega^{2}
$$

where $d \omega^{2}$ is the line element of a unit $(n-2)$-sphere. A natural choice for the boundary, $\mathcal{T}$, of the manifold is the history of a $(n-2)$-sphere of constant radial coordinate $r=r_{\mathrm{B}}$. (We will neglect the initial and final spacelike boundary pieces.) A solution to the conformally related theory $2.1 \mathrm{~b})$ is

$$
d \tilde{s}^{2}=-\Omega^{2} N^{2}(r) d t^{2}+\frac{d r^{2}}{\Omega^{-2} f^{2}(r)}+\Omega^{2} r^{2} d \omega^{2} .
$$

We find that $k_{\text {flat }}=-(n-2) / r_{\mathrm{B}}$ and $\tilde{k}_{\text {flat }}=-(n-2) /\left(\Omega r_{\mathrm{B}}\right)$. Thus, we see that the boundary action functionals given in (2.8) suitably transform into each other. 
The quasilocal mass [3:7] is a charge associated with a timelike Killing vector on the boundary manifold $\mathcal{T}$. Unlike the quasilocal energy defined below, the quasilocal mass is conserved in the sense that it does not depend upon any foliation of the boundary manifold $\mathcal{T}$; in the absence of matter flux through $\mathcal{T}$, the value of the quasilocal mass does not change with time. For a sss spacetime solution of the form (2.9) to the theory given by the action (2.1a) with background action (2.8a), the quasilocal mass is 四

$$
\begin{aligned}
M(r)=2 N(r)\left(\frac{(n-2) A_{n-2}(r) D(\phi)}{r}\right. & \\
& \left.-f(r) \frac{d}{d r}\left(A_{n-2}(r) D(\phi)\right)\right)
\end{aligned}
$$

where $A_{m}(r)$ is the surface "area" of an $m$-sphere of radius $r$ :

$$
A_{m}(r)=\frac{(4 \pi)^{m / 2} \Gamma(m / 2)}{\Gamma(m)} r^{m} .
$$

The mass of a sss solution to the theory given by the action (2.1b) with background action (2.8b) is

$$
\begin{aligned}
\tilde{M}(r)=2 \tilde{N}(r)( & \frac{(n-2) \tilde{A}_{n-2}(r) \tilde{D}(\tilde{\phi})}{\Omega r} \\
& \left.-\tilde{f}(r) \frac{d}{d r}\left(\tilde{A}_{n-2}(r) \tilde{D}(\tilde{\phi})\right)\right) .
\end{aligned}
$$

It is easily verified that $\tilde{M}(r)=M(r)$ if the solutions are related by the conformal tranformation of eq. (2.2) since $\tilde{A}_{n-2}(r)=\Omega^{n-2} A_{n-2}(r)$.

Although the background action of eq. (2.8a) has many attractive features, there are several limitations to the notion of mass given by eq. (2.11). Because we chose the background action functional to be related to the extrinsic curvature of the boundary metric embedded in flat space, the definition is only suited to solutions that are asymptotically flat. However, the notion of mass can be easily generalized by replacing $k_{\text {flat }}$ with the trace of the extrinsic curvature of the $(n-2)$-surface $\mathcal{T} \cap \Sigma_{t}$ embedded in a spacelike slice of a sss spacetime with appropriate asymptotic behavior that is considered to be a reference spacetime (if such an embedding is possible). For spacetimes that are not spherically symmetric, it is not clear whether the background action functional of eq. (2.8a) will have the appropriate conformal tranformation property.

The ADM mass is defined as the value of the gravitational Hamiltonian evaluated on a surface at spacelike infinity in an asymptotically flat spacetime. That this definition of ADM mass agrees with the original definition [8] has been demonstrated in Refs. 9. [10] for General Relativity. A similar analysis shows that the Hamiltonian for the gravitational action of eq. (2.1a) is the same as the integral over a spacelike hypersurface of the time-time component of the metric equation of motion. However, for the sss metric of eq. (2.9), there is a disagreement between the quasilocal mass and the above notion of an ADM mass: If the conformal transformation does not approach unity in the asymptotically flat regime, the asymptotic value of the lapse function in one solution will not be the same as the asymptotic value of the lapse in the conformally related solution. In the ADM prescription, we would 
renormalize the time variable (since the solution is static) so that the lapse approaches unity as the radius approaches infinity. This will cause the two ADM masses to differ by a constant factor equal to the asymptotic value of the conformal factor.

Rather than choosing the background action functional of eq. (2.8a), one may choose the background action along the lines proposed by Hawking and Horowitz [10]: the reference action functional $I_{0}$ has the same functional form as the rest of the action $I$, but is a functional of the variables $\left(g_{\mathrm{ref}}\right)_{a b}$ and $\phi_{\mathrm{ref}}$, which are independent of $g_{a b}$ and $\phi$ except on the boundary. On the boundary we require $\left(\gamma_{\mathrm{ref}}\right)_{a b} \equiv \gamma_{a b}$ and $\phi_{\text {ref }} \equiv \phi$. The reference spacetime is the solution to the field equations produced from the action $I_{0}$. Under this prescription, the mass of the sss solution (2.9) is

$$
M(r)=2 N(r)\left(f_{\mathrm{ref}}(r)-f(r)\right) \frac{d}{d r}\left(A_{n-2}(r) D(\phi)\right)
$$

where the reference spacetime solution has the same form as eq. (2.9). Notice that $N_{\text {ref }} \equiv N$ and $\phi_{\text {ref }} \equiv \phi$ since these are fields on the boundary manifold, while $f_{\text {ref }}$ is independent of $f$. Typically, Minkowski spacetime will be a Sss solution to the field equations generated by the reference action functional; if this is chosen as the reference spacetime, then we have $f_{\text {ref }}=1$ in eq. (2.14). Although the reference action functional $I_{0}$ is conformally invariant (provided the requirements of the previous subsection are satisfied), the mass of eq. (2.14) will not be conformally invariant because one would naturally choose the same reference solution - rather than the conformal transform of the reference solution - in the conformally transformed theory.

Nevertheless, there seem to be situations in which the mass defined by eq. (2.14) is more attractive than the conformally invariant mass of eq. (2.11). For example, in the asymptotically anti-de Sitter $(2+1)$-dimensional black hole solution of Martínez and Zanelli [11], the mass obtained from eq. (2.14) is finite (and agrees with the result of Martínez and Zanelli) as $r \rightarrow \infty$, while the mass obtained from eq. (2.11) linearly diverges as $r \rightarrow \infty$. Transforming to the "Einstein frame" (i.e., the form of the action with constant $D$ ), we find that the mass has the same divergent behaviour, as expected since (2.11) was chosen to be conformally invariant. In the Einstein frame, the two prescriptions are equivalent, so the mass obtained from (2.14) likewise diverges.

We have shown that the quasilocal mass of a sss solution of dilaton gravity is conformally invariant provided that a suitable background action functional can be chosen. However, we should note that the quasilocal energy, representing the thermodynamic internal energy of the gravitating system, cannot be conformally invariant for the following reason: although the entropy, $S$, and the surface gravity of a black hole are conformally invariant, the temperature of a black hole, $T$, as measured on the finite-sized quasilocal surface, is not conformally invariant because of the presence of the blue-shift factor. Thus, the thermodynamic internal energy, which must satisfy the first law of thermodynamics, $T d S=d E+$ work terms, cannot be conformally invariant either. In fact, conformal invariance of thermodynamic quantities should not be expected because these quantites are observer-dependent (although the laws of thermodynamics are not); we discuss the thermodynamic variables in the next section. 


\section{THERMODYNAMIC VARIABLES}

Consider a dilaton theory of gravity given by the action of eq. (2.1a) with an additional Maxwell-dilaton interaction:

$$
I_{\mathrm{M}}=\int_{\mathcal{M}}^{{ }^{n} \boldsymbol{\epsilon}} \frac{1}{4} W(\phi) \mathfrak{F}^{a b} \mathfrak{F}_{a b}
$$

where $\mathfrak{F}=\boldsymbol{d} \mathfrak{A}$ is the two-form strength of the Maxwell field with one-form potential $\mathfrak{A}$. In Ref. [6], it was shown that the first law of thermodynamics for classical sss black hole solutions to such a theory is

$$
\begin{aligned}
\delta S=\int_{\overline{\mathcal{T}}} N \boldsymbol{d} \tau \wedge\left(\delta \boldsymbol{E}+\mathcal{S} \delta^{(n-2)} \boldsymbol{\epsilon}\right. \\
\left.+\boldsymbol{\mu} \delta \phi+\mathfrak{V} \delta \mathfrak{Q}+\mathfrak{K}^{a} \delta \mathfrak{W}_{a}\right) .
\end{aligned}
$$

Here, $N=\left(u^{a} \nabla_{a} \tau\right)^{-1}$ represents the lapse function associated with the foliation of the spacetime into spatial hypersurfaces of constant Euclidean time $\tau$ and timelike normal $u^{a}$. The foliation is degenerate at the event horizon of the black hole; the Euclidean manifold must be periodic in time with period $\Delta \tau=2 \pi / \kappa_{\mathrm{H}}\left(\kappa_{\mathrm{H}}\right.$ is the surface gravity of the event horizon) in order to avoid a conical singularity at the event horizon. The manifold $\overline{\mathcal{T}}$ is the periodic history of a $(n-2)$-sphere quasilocal surface $\mathcal{B}$ with volume element ${ }^{(n-2)} \boldsymbol{\epsilon}$ and radius $r_{\mathrm{B}} . \boldsymbol{E}, \boldsymbol{\mu}, \mathfrak{Q}$, and $\mathfrak{K}^{a}$ are $(n-2)$-forms representing the quasilocal surface energy, dilaton potential, Maxwell charge, and Maxwell current densities respectively. $\mathcal{S}$ is the quasilocal surface tension, while $\mathfrak{V}=-u^{a} \mathfrak{A}_{a}$ and $\mathfrak{W}_{a}=\sigma_{a}^{b} \mathfrak{A}_{b}$ with $\sigma_{a b}=\gamma_{a b}+u_{a} u_{b}$. The entropy of the black hole is given by

$$
S=4 \pi \int_{\mathcal{H}}{ }^{(n-2)} \boldsymbol{\epsilon} D(\phi)=\left.4 \pi A_{n-2}\left(r_{\mathrm{H}}\right) D(\phi)\right|_{\phi=\phi\left(r_{\mathrm{H}}\right)}
$$

where $\mathcal{H}$ is the event horizon $(n-2)$-sphere with radius $r_{\mathrm{H}}$, and $A_{n-2}\left(r_{\mathrm{H}}\right)$ is the surface "area" of the event horizon given by eq. (2.12). Given the conformal transformation properties of the area and the dilaton function $D(\phi)$ stated in the previous section, we see that the entropy is conformally invariant.

Because the solution is static, the $\tau$ integral yields an overall factor of $\beta=N \Delta \tau$, which is the inverse temperature on the quasilocal surface. The quasilocal energy, dilaton potential, and Maxwell charge can be calculated as follows [6]:

$$
\begin{gathered}
E=\int_{\mathcal{B}} \boldsymbol{E}=-2 A\left(n^{a} \nabla_{a} D(\phi)-D(\phi) k\right)-E_{0} \\
\mu=\int_{\mathcal{B}} \boldsymbol{\mu}=2 A\left(H(\phi) n^{a} \nabla_{a} \phi\right. \\
\left.-(d D / d \phi)\left(k-n^{a} a_{a}\right)\right)-\mu_{0} \\
\mathfrak{Q}=\int_{\mathcal{B}} \mathfrak{Q}=-A W(\phi) n^{a} \mathfrak{E}_{a}-\mathfrak{Q}_{0} .
\end{gathered}
$$

Here, $a^{b}=u^{a} \nabla_{a} u^{b}$ is the acceleration of the normal vector $u^{a}$ (i.e., the acceleration of an observer on the quasilocal surface), $k_{a b}=-\frac{1}{2} £_{n} \sigma_{a b}$ is the extrinsic curvature of the quasilocal 
surface as embedded in a spacelike leaf of the foliation, and $\mathfrak{E}_{a}=\mathfrak{F}_{a b} u^{b}$ is the electric field strength. $A=A_{n-2}\left(r_{\mathrm{B}}\right)$ is the "area" of the $(n-2)$-sphere $\mathcal{B}$ given by eq. (2.12). The Maxwell surface current density, $\mathfrak{K}^{a}$, should not contribute to the first law of thermodynamics in the spherically symmetric case as there is no preferred direction on the quasilocal surface $\mathcal{B}$. However, we will need it in order to calculate the electromagnetic work term for magnetic monopoles; the surface current density is given by

$$
\mathfrak{K}^{a}=-{ }^{(n-2)} \boldsymbol{\epsilon} W(\phi) \mathfrak{F}_{c d} \sigma^{a c} n^{d}-\mathfrak{K}_{0}^{a} .
$$

The surface tension is given by [6, [7]

$$
\mathcal{S}=2 n^{a} \nabla_{a} D(\phi)+2 D(\phi)\left(n^{a} a_{a}-k\left(\frac{n-3}{n-2}\right)\right)-\mathcal{S}_{0} .
$$

Because of spherical symmetry, we can re-write eq. (3.2) in the differential form

$$
T d S=d E+\mathcal{S} d A+\mu d \phi+\mathfrak{V} d \mathfrak{Q}
$$

where $T=\beta^{-1}$ is the Hawking temperature blue-shifted to its observed value on the quasilocal surface.

Each quantity in Eqs. (3.4)-(3.8) includes a term (with a subscripted "0") that arises from the background action functional $I_{0}$. We shall assume that this action functional does not depend on the Maxwell field so that $\mathfrak{Q}_{0}$ and $\mathfrak{K}_{0}^{a}$ both vanish. We further require the background action to be a linear functional of the lapse $N$ [3]. Then

$$
E_{0}=-\int_{\mathcal{B}}\left(\delta I_{0} / \delta N\right)
$$

is the reference energy [3]; the reference surface tension and dilaton potential can be calculated from the reference energy via $\mathcal{S}_{0}=-\left(\partial E_{0} / \partial A\right)$ and $\mu_{0}=-\left(\partial E_{0} / \partial \phi\right)$ 《.

\section{THE GHS SOLUTION}

The GHS solution [1,2] in the "string frame" is a solution of equations of motion arising from the action

$$
\begin{gathered}
I=\frac{1}{16 \pi} \int_{\mathcal{M}}{ }^{4} \boldsymbol{\epsilon} \mathrm{e}^{-2 \phi}\left(R+4(\nabla \phi)^{2}-\mathfrak{F}^{a b} \mathfrak{F}_{a b}\right) \\
-\frac{1}{8 \pi} \int_{\mathcal{T}}{ }^{3} \boldsymbol{\epsilon} \mathrm{e}^{-2 \phi} \Theta-I_{0} .
\end{gathered}
$$

We are interested in two solutions of the equations of motion generated by this action: the electrically charged and the magnetically charged black hole solution. The magnetic string

black hole solution is [1]

$$
\mathrm{e}^{-2 \phi}=\mathrm{e}^{-2 \phi_{0}}\left(1-\frac{q^{2}}{a r}\right)
$$




$$
\begin{gathered}
\mathfrak{F}=q \boldsymbol{d} \vartheta \wedge \sin \vartheta \boldsymbol{d} \varphi \\
f^{2}(r)=\left(1-\frac{2 a}{r}\right)\left(1-\frac{q^{2}}{a r}\right)
\end{gathered}
$$

and

$$
N(r)=f(r) \mathrm{e}^{2\left(\phi-\phi_{0}\right)}
$$

where the line element is given by eq. (2.9); $\phi_{0}, q$, and $a$ are constants of integration.2 This black hole has an event horizon at $r_{\mathrm{H}}=2 a$. The electric string black hole solution is [2]

$$
\begin{gathered}
\mathrm{e}^{-2 \phi}=\mathrm{e}^{2 \phi_{0}}+\frac{q^{2}}{a r} \\
\mathfrak{F}=-\frac{q \mathrm{e}^{2 \phi}}{r^{2}} N(r) \boldsymbol{d} t \wedge f^{-1}(r) \boldsymbol{d} r \\
f^{2}(r)=1+\mathrm{e}^{-2 \phi_{0}}\left(\frac{q^{2}-2 a^{2}}{a r}\right)
\end{gathered}
$$

and

$$
N(r)=f(r) \mathrm{e}^{2\left(\phi+\phi_{0}\right)}
$$

The event horizon for this black hole solution is at $r_{\mathrm{H}}=\mathrm{e}^{-2 \phi_{0}}\left(2 a-q^{2} / a\right)$. For both of these solutions, the quasilocal mass can be computed with eq. (2.11) if we assume that the appropriate choice of the background action functional is made. We find $\lim _{r \rightarrow \infty} M(r)=$ $a$ for the electric solution and $a \mathrm{e}^{-2 \phi_{0}}$ for the magnetic solution.

Using the conformal transformation (1.3) with the conformal factor given by eq. (4.2a) and eq. (4.3a) for the magnetic and electric solutions respectively, we recover the solution in the "Einstein frame." Let $\varrho=r \mathrm{e}^{-\phi}$; the metric in the Einstein frame then yields

$$
d s^{2}=-N^{2} \mathrm{e}^{-2 \phi} d t^{2}+\frac{d \varrho^{2}}{f^{2} \mathrm{e}^{2 \phi}(d \varrho / d r)^{2}}+\varrho^{2} d \omega^{2}
$$

for either the magnetic or electric cases. This is a solution of the theory given by the action in the Einstein frame:

$$
\begin{gathered}
I=\frac{1}{16 \pi} \int_{\mathcal{M}}{ }^{4} \boldsymbol{\epsilon}\left(R-2(\nabla \phi)^{2}-\mathrm{e}^{-2 \phi} \mathfrak{F}^{a b} \mathfrak{F}_{a b}\right) \\
-\frac{1}{8 \pi} \int_{\mathcal{T}}{ }^{3} \boldsymbol{\epsilon} \Theta-I_{0} .
\end{gathered}
$$

\footnotetext{
${ }^{2}$ The constant of integration, $a$, is the combination of constants $m \mathrm{e}^{\phi_{0}}$ used in Refs. [1],2].
} 
If the background action function is chosen appropriately, then eq. (2.11) can be used to calculate the ADM mass of the solution (4.4) for both the electric and the magnetic cases. We find $\lim _{\varrho \rightarrow \infty} M(\varrho)=a$ for the electric solution and $a \mathrm{e}^{-2 \phi_{0}}$ for the magnetic solution. As expected, these masses agree with those we obtained from the metric in the string frame. However, when $\phi_{0} \neq 0$, the conformal transformation does not approach unity at spatial infinity, and thus the lapse function $\mathrm{Ne}^{-\phi}$ of eq. (4.4) also fails to approach unity at spatial infinity. Since the solution is static, we can rescale the time coordinate: $t \rightarrow t_{\text {new }}=\mathrm{e}^{ \pm \phi_{0}}$ where the plus (minus) applies to the magnetic (electric) solution. The new lapse function is $N \mathrm{e}^{-\left(\phi \mp \phi_{0}\right)}$. The mass rescales by a constant factor, and the result $M_{\mathrm{ADM}}=a \mathrm{e}^{-\phi_{0}}$ is obtained for both the magnetic and electric solutions, in agreement with Refs. [1, 2]. We note that such a rescaling is always possible for static solutions and, unless there is some required asymptotic behaviour for the lapse function, the definition of mass contains an arbitrary constant factor.

Consider now the mass defined by eq. (2.14) with the reference solution of Minkowski spacetime with a constant dilaton. In the Einstein frame, the mass of eq. (2.14) agrees with the mass of eq. (2.11) because $D$ is constant. However, in the string frame, eq. (2.14) yields $M=\left(a-q^{2} /(2 a)\right)$ for the electric solution and $M=\mathrm{e}^{-2 \phi_{0}}\left(a+q^{2} /(2 a)\right)$ for the magnetic solution. Clearly the mass is not conformally invariant when eq. (2.14) is used.

\section{A. Thermodynamics of the Electric String Solution}

The electric string solution is given by Eqs. (4.3). We can evaluate the extensive thermodynamic variables using Eqs. (3.3), (3.4), and (3.6). We find

$$
S=2 \pi a r_{\mathrm{B}}\left(\frac{2 a^{2}-q^{2}}{a r_{\mathrm{B}} \mathrm{e}^{-2 \phi}-q^{2}}\right)
$$

is the entropy of the black hole,

$$
E=-f\left(r_{\mathrm{B}}\right)\left(r_{\mathrm{B}} \mathrm{e}^{-2 \phi}-\frac{q^{2}}{2 a}\right)-E_{0}
$$

is the quasilocal energy, and

$$
\mathfrak{Q}=q
$$

is the Maxwell charge. Note that we can solve eq. (4.6) for the parameter $a$ to obtain an expression for $a$ in terms of the extensive variables $S, \mathfrak{Q}, \phi$, and $A=4 \pi r_{\mathrm{B}}^{2}$. We can write $f\left(r_{\mathrm{B}}\right)$ in terms of these extensive variables and, thus, write $E=E(S, A, \phi, \mathfrak{Q})$. The zero of the energy, $E_{0}$, is set by the background action functional via (3.10).

The intensive variables include the temperature, the surface tension, the dilaton potential, and the Maxwell potential. The temperature is $T=\kappa_{\mathrm{H}} /(2 \pi N)$ with $\kappa_{\mathrm{H}}=f(d N / d r)$ evaluated on the event horizon. We find

\footnotetext{
${ }^{3}$ In fact, the mass in the Einstein frame and in the string frame agree for a quasilocal boundary at any radius, not just at spacelike infinity.
} 


$$
T=\frac{1}{8 \pi a N\left(r_{\mathrm{B}}\right)}\left(\mathrm{e}^{-2 \phi}-\frac{q^{2}}{a r_{\mathrm{B}}}\right) .
$$

Using eq. (3.8), we compute the surface tension,

$$
\mathcal{S}=\frac{1}{8 \pi r_{\mathrm{B}}^{2}}\left(f\left(r_{\mathrm{B}}\right)\left(r_{\mathrm{B}} \mathrm{e}^{-2 \phi}-\frac{q^{2}}{2 a}\right)+\frac{a}{f\left(r_{\mathrm{B}}\right)}\right)-\mathcal{S}_{0} .
$$

We use eq. (3.5) to calculate the dilaton potential:

$$
\mu=-f\left(r_{\mathrm{B}}\right)\left(2 r_{\mathrm{B}} \mathrm{e}^{-2 \phi}-\frac{q^{2}}{2 a}\right)-\frac{a}{f\left(r_{\mathrm{B}}\right)}-\mu_{0} .
$$

The contributions of the reference spacetime to the surface tension and dilaton potential, $\mathcal{S}_{0}$ and $\mu_{0}$, are determined in terms of $E_{0}$ as stated at the end of Sec. III. Finally, the Maxwell potential, $\mathfrak{V}$, is given by [6]

$$
\mathfrak{V}=\frac{1}{N\left(r_{\mathrm{B}}\right)} \int_{r_{\mathrm{H}}}^{r_{\mathrm{B}}} \mathfrak{F}_{t r} d r=-\frac{q}{2 a} f\left(r_{\mathrm{B}}\right)
$$

Notice that $\mathfrak{V}$ is finite on the event horizon.

We can express the quasilocal energy (4.7) in terms of the extensive variables $S, A, \phi$, and $\mathfrak{Q}$ using (4.6) and (4.8). It can then be shown that

$$
T=\frac{\partial E}{\partial S}, \quad \mathcal{S}=-\frac{\partial E}{\partial A}, \quad \mu=-\frac{\partial E}{\partial \phi}, \quad \text { and } \quad \mathfrak{V}=-\frac{\partial E}{\partial \mathfrak{Q}} .
$$

Thus, the quasilocal energy is the appropriate choice for the thermodynamic internal energy of the gravitating system contained within the quasilocal boundary; we have explicitly demonstrated the first law of thermodynamics given by eq. (3.9). This thermodynamic relation is independent of the choice of the background action functional $I_{0}$ and, thus, independent of the choice of $E_{0}$.

Let us examine the asymptotic behaviour of the thermodynamic variables for large values of $r_{\mathrm{B}}$ or, equivalently, for small values of $u=1 / r_{\mathrm{B}}$. The Maxwell charge, $\mathfrak{Q}=q$, and the entropy, $S=2 \pi \mathrm{e}^{-2 \phi_{0}}\left(2 a^{2}-q^{2}\right)$, are both independent of the size of the quasilocal system. The quasilocal energy has the following behaviour:

$$
E=\left\{-\mathrm{e}^{2 \phi_{0}} u^{-1}+\left(a-\frac{q^{2}}{a}\right)+O(u)\right\}-E_{0} .
$$

Unless $E_{0}$ is suitably chosen, the quasilocal energy will diverge as $r_{\mathrm{B}} \rightarrow \infty$. The temperature,

$$
T=\frac{1}{8 \pi a}\left\{\mathrm{e}^{2 \phi_{0}}+\left(a+\frac{q^{2}}{2 a}\right) u+O\left(u^{2}\right)\right\}
$$

approaches the value $\mathrm{e}^{2 \phi_{0}} /(8 \pi a)$ as $r_{\mathrm{B}} \rightarrow \infty$, and the Maxwell potential,

$$
\mathfrak{V}=-\frac{q}{2 a}\left\{1+\mathrm{e}^{-2 \phi_{0}}\left(q^{2} / 2-a^{2}\right) u+O\left(u^{2}\right)\right\},
$$


approaches $-q /(2 a)$. The surface tension and the dilaton potential have the following behaviour:

$$
\mathcal{S}=\frac{1}{8 \pi}\left\{\mathrm{e}^{2 \phi_{0}} u+\frac{q^{2}}{a} u^{2}+O\left(u^{3}\right)\right\}+\left(\frac{\partial E_{0}}{\partial A}\right)
$$

and

$$
\mu=\left\{-2 \mathrm{e}^{2 \phi_{0}} u^{-1}+\left(a-\frac{5 q^{2}}{2 a}\right)+O(u)\right\}+\left(\frac{\partial E_{0}}{\partial \phi}\right)
$$

respectively. As with the energy, the asymptotic behaviour of these functions depends upon the choice of background action functional; in particular, the dilaton potential will diverge as $r_{\mathrm{B}} \rightarrow \infty$ unless a suitable choice for $E_{0}$ is made. We will consider two possible choices of $I_{0}$ and calculate the asymptotic behaviour of the energy, the surface tension, and the dilaton potential. (In the trivial case $I_{0}=0$, we see that the energy and the dilaton potential both diverge as $r_{\mathrm{B}} \rightarrow \infty$.)

Our choice of $I_{0}$ is the functional of eq. (2.8a). We find $E_{0}=r_{\mathrm{B}} \mathrm{e}^{-2 \phi}, \mu_{0}=2 r_{\mathrm{B}} \mathrm{e}^{-2 \phi}$, and $\mathcal{S}_{0}=-\mathrm{e}^{-2 \phi} /\left(8 \pi r_{\mathrm{B}}\right)$. The quasilocal energy is finite in the limit $r_{\mathrm{B}} \rightarrow \infty$ and approaches the value $a$; the dilaton potential is also finite and approaches $q^{2} /(2 a)$ in this limit; the surface tension, $\mathcal{S} \sim O\left(u^{3}\right)$ for large values of $r_{\mathrm{B}}$.

\section{B. Thermodynamics of the Magnetic String Solution}

Recall now the magnetic string solution of Eqs. (4.2). We wish to construct the thermodynamic variables in the same way as we have just done for the electric string solution. The entropy and the energy can both be calculated as before. They are

$$
S=2 \pi a r_{\mathrm{B}} \mathrm{e}^{-2 \phi}\left(\frac{2 a^{2}-q^{2}}{a r_{\mathrm{B}}-q^{2}}\right)
$$

and

$$
E=-\mathrm{e}^{-2 \phi}\left(f\left(r_{\mathrm{B}}\right) r_{\mathrm{B}}+N\left(r_{\mathrm{B}}\right) \frac{q^{2}}{2 a}\right)-E_{0}
$$

respectively. We can also calculate the following intensive variables as before: The temperature is

$$
T=\frac{1}{8 \pi a N\left(r_{\mathrm{B}}\right)},
$$

the surface tension is

$$
\mathcal{S}=\frac{1}{8 \pi r_{\mathrm{B}}} \mathrm{e}^{-2 \phi}\left(f\left(r_{\mathrm{B}}\right) r_{\mathrm{B}}+N\left(r_{\mathrm{B}}\right) \frac{q^{2}}{2 a}+\frac{a}{N\left(r_{\mathrm{B}}\right)}\right)-\mathcal{S}_{0},
$$

and the dilation potential is

$$
\mu=-\mathrm{e}^{-2 \phi}\left(2 f\left(r_{\mathrm{B}}\right) r_{\mathrm{B}}+N\left(r_{\mathrm{B}}\right) \frac{q^{2}}{2 a}+\frac{a}{N\left(r_{\mathrm{B}}\right)}\right)-\mu_{0} .
$$


There is a difficulty in evaluating the electromagnetic work term. It is not possible to produce the Maxwell field strength of eq. (1.2b) with a non-singular potential $\mathfrak{A}$. For example, the potential $\mathfrak{A}=q(1-\cos \vartheta) \boldsymbol{d} \varphi$ has a Dirac string singularity at $\vartheta=\pi$. Because the terms in eq. (3.2) that give rise to the Maxwell work terms involve projections of the potential, $\mathfrak{A}$, it is necessary to invoke some trick to avoid the Dirac string singularity in the surface integral. Let $\delta Q_{\mathrm{M}}$ denote the element of work from the Maxwell field. From eq. (3.2), we have

$$
\beta \delta Q_{\mathrm{M}}=\int_{\overline{\mathcal{T}}} N \boldsymbol{d} \tau \wedge \mathfrak{K}^{a} \delta \mathfrak{W}_{a}
$$

where the " $\mathfrak{V} \delta \mathfrak{Q}$ " term does not appear because $\mathfrak{Q}$ vanishes for purely spatial Maxwell field strength two-forms. The topology of the Euclidean manifold, $\overline{\mathcal{M}}$, is $D^{2} \times S^{2}$ where $D^{2}$ is a two dimensional disk and $S^{2}$ is a two-sphere. Consider instead the Euclidean manifold $\hat{\mathcal{M}}$ with topology $D^{2} \times S_{\theta}^{2}$ where $S_{\theta}^{2}$ is the fragment of a sphere $\vartheta \in[0, \theta)$ where $\theta<\pi$. The boundary of this manifold, $\hat{\mathcal{T}}$, consists of the (periodic) history of the sphere fragment $S_{\theta}^{2}$ of radius $r_{\mathrm{B}}$, and the (periodic) history of the cone, $C_{\theta}^{2}$, of constant $\vartheta=\theta$ and radius running from the event horizon $r_{\mathrm{H}}$ to the boundary $r_{\mathrm{B}}$. Because the solution is static, we find

$$
\beta \delta \hat{Q}_{\mathrm{M}}=\Delta \tau\left(\int_{S_{\theta}^{2}} N \mathfrak{K}^{a} \delta \mathfrak{W}_{a}+\int_{C_{\theta}^{2}} N \mathfrak{K}^{a} \delta \mathfrak{W}_{a}\right) .
$$

Notice that the integral now avoids the Dirac string singularity.

Using eq. (3.7) we see that $\mathfrak{K}^{a}=0$ on $S_{\theta}^{2}$. However, on $C_{\theta}^{2}$, we have

$$
\mathfrak{K}^{\varphi}={ }^{2} \boldsymbol{\epsilon} \frac{\mathrm{e}^{-2 \phi} q}{4 \pi r^{3} \sin \theta}
$$

and

$$
\mathfrak{W}_{\varphi}=q(1-\cos \theta) .
$$

The volume element on $C_{\theta}^{2}$ can be written ${ }^{2} \boldsymbol{\epsilon}=-f^{-1}(r) \boldsymbol{d} r \wedge r \sin \theta \boldsymbol{d} \varphi$ where the negative sign arises because the unit normal, $n^{a}=r^{-1}(\partial / \partial \vartheta)^{a}$, points inwards. eq. (4.25) can be computed:

$$
\beta \delta \hat{Q}_{\mathrm{M}}=\beta\left(\frac{1-\cos \theta}{2}\right)\left(\frac{q \mathrm{e}^{-2 \phi}}{f\left(r_{\mathrm{B}}\right)}\left(\frac{1}{r_{\mathrm{B}}}-\frac{1}{r_{\mathrm{H}}}\right)\right) \delta q .
$$

Now we assume that $Q_{\mathrm{M}}=\lim _{\theta \rightarrow \pi} \hat{Q}_{\mathrm{M}}$. Furthermore, we write $Q_{\mathrm{M}}=\mathfrak{V} \delta \mathfrak{Q}$ and, thus, define

$$
\mathfrak{Q}=q
$$

and

$$
\mathfrak{V}=\mathrm{e}^{-2 \phi} \frac{q}{f\left(r_{\mathrm{B}}\right)}\left(\frac{1}{r_{\mathrm{B}}}-\frac{1}{r_{\mathrm{H}}}\right) .
$$

The quasilocal energy can be written in terms of the extensive variables $S, A, \phi$, and $\mathfrak{Q}$. Then the relations of eq. (4.13) can be shown to hold. Thus, the definition of the thermodynamic variables are consistent with the first law of thermodynamics (3.9). 
The entropy, $S=2 \pi \mathrm{e}^{-2 \phi_{0}}\left(2 a^{2}-q^{2}\right)$, and Maxwell charge, $\mathfrak{Q}=q$, are independent of the size, $r_{\mathrm{B}}$, of the quasilocal region. For $r_{\mathrm{B}} \rightarrow \infty, T \rightarrow(8 \pi a)^{-1}$, and $\mathfrak{V} \rightarrow-\mathrm{e}^{-2 \phi_{0}} q /(2 a)$. Finally, we demonstrate the asymptotic behaviour of the energy, the surface tension, and the dilaton force for small values of $u=1 / r_{\mathrm{B}}$. We find

$$
\begin{gathered}
E=\mathrm{e}^{-2 \phi_{0}}\left\{u^{-1}+\left(a+\frac{q^{2}}{a}\right)+O(u)\right\}-E_{0} \\
\mathcal{S}=\frac{1}{8 \pi} \mathrm{e}^{-2 \phi_{0}}\left\{u-\frac{q^{2}}{a} u^{2}+O\left(u^{3}\right)\right\}+\left(\frac{\partial E_{0}}{\partial A}\right)
\end{gathered}
$$

and

$$
\mu=\mathrm{e}^{-2 \phi_{0}}\left\{-2 u^{-1}+\left(a+\frac{5 q^{2}}{2 a}\right)+O(u)\right\}+\left(\frac{\partial E_{0}}{\partial \phi}\right)
$$

respectively. For the background action functional of eq. (2.8a), we have $E_{0}=-\mathrm{e}^{-2 \phi} u^{-1}$. Then the quasilocal energy approaches the value $\mathrm{e}^{-2 \phi_{0}} a$ as $r_{\mathrm{B}} \rightarrow \infty$, and the dilaton potential approaches $\mathrm{e}^{-2 \phi_{0}}\left(a+q^{2} /(2 a)\right)$. For large $r_{\mathrm{B}}, \mathcal{S} \sim O\left(u^{3}\right)$.

\section{SUMMARY}

We have shown that conformal invariance of conserved quantities such as the mass is not automatically guaranteed: it crucially depends on the choice of the background action functional. We have presented a background action functional that yields a conformally invariant quasilocal mass for static, spherically symmetric spacetimes, but it is not clear if this conformal invariance will continue to hold for more general solutions. This quasilocal mass evaluated as $r \rightarrow \infty$ for asymtotically flat spacetimes will recover the ADM mass up to a constant factor. There is no guarantee that this conformally invariant mass is finite, as illustrated by the non-asymptotically flat solution of Ref. [11. We have also presented an alternate choice of reference action functional based on the one suggested by Hawking and Horowitz [10]. However, the resulting mass is not conformally invariant.

We have illustrated the calculation of the above masses for the Garfinkle, Horowitz, and Strominger [1.2] static, spherically symmetric string black hole solutions. We found that the quasilocal mass with our proposed background action functional is conformally invariant, but that the mass arising from the action with the reference given by the prescription of Hawking and Horowitz is not. In addition, we calculated the internal energy and other thermodynamic variables for these solutions. For the magnetically charged black hole solution, we adopted a trick to avoid the Dirac string singularity. The first law of thermodynamics is found to hold for these thermodynamic variables. Although the individual thermodynamic variables depend on the choice of the background action funtional, the first law of thermodynamics does not. Also, the first law of thermodynamics is conformally invariant even though the individual thermodynamic variables are not.

\section{ACKNOWLEDGMENTS}

This work has been supported in part by the Natural Sciences and Engineering Research Council (NSERC) of Canada. 


\section{REFERENCES}

[1] D. Garfinkle, G. T. Horowitz, and A. Strominger, Phys. Rev. D 433140 (1991); erratum, 453888 (1992).

[2] G. T. Horowitz, preprint hep-th/9210119 (1992).

[3] J. D. Brown and J. W. York, Phys. Rev. D 471407 (1993).

[4] J. D. E. Creighton and R. B. Mann, Phys. Rev. D 524569 (1995).

[5] R. B. Mann, Phys. Rev. D 474438 (1993).

[6] J. D. E. Creighton and R. B. Mann, preprint gr-qc/9511012 (1995).

[7] J. D. Brown, J. D. E. Creighton, and R. B. Mann, Phys. Rev. D 506394 (1994).

[8] R. Arnowitt, S. Deser, and C. Misner, in Gravitation: An Introduction to Current Research, ed. L. Witten (New York, Wiley, 1962).

[9] V. Iyer and R. M. Wald, Phys. Rev. D 50846 (1994).

[10] S. W. Hawking and G. T. Horowitz, preprint gr-qc/9501014 (1995).

[11] C. Martínez and J. Zanelli, preprint gr-qc/9604021 (1996). 